\title{
Una experiencia de grupos terapéuticos con adolescentes y jóvenes en un centro de salud mental
}

RESUMEN: El presente trabajo describe la experiencia de tratamiento grupal con adolescentes y jóvenes desarrollada en un Centro de Salud Mental.

PALABRAS CLAVE: adolescencia, juventud, tratamiento grupal, identidad, maduración.
SUMMARY: This work describes our group treatment experience with teenagers and youth developed in a Mental Health Center.

KEY WORDS: adolescence, youth, group treatment, identity, maturing.

\section{Introducción}

La adolescencia es una etapa importante en el desarrollo del individuo, supone un proceso gradual de maduración que conduce al acceso a la edad adulta (1). El adolescente o joven debe dar respuesta a cuestiones tan importantes como su propia identidad, su imagen corporal y su madurez sexual, la relación con sus padres, con sus iguales y sus relaciones afectivas. Las relaciones sociales y afectivas que establece le permiten ensayar, a través de las identificaciones que realiza, cuáles son sus nuevas capacidades, las expectativas que tiene sobre sí mismo y contribuyen a la elaboración de su identidad adulta. Dada la importancia del grupo en este momento vital y por el efecto movilizador y constructivo que tienen los tratamientos grupales, hemos desarrollado este tipo de intervención terapéutica con adolescentes que acuden a nuestro centro de trabajo.

Son muchos los motivos por los que los grupos terapéuticos son una buena alternativa de tratamiento. En términos económicos suponen menores costes tanto en tiempo como en especialistas, lo cual es de vital importancia en un contexto como el de la salud pública, en el que la presión asistencial muchas veces dificulta el acceso a los recursos. Pero además, desde sus inicios, los grupos terapéuticos han acreditado su eficacia en múltiples ocasiones. Burlingame y cols. (2) realizaron en 1995 un estudio de meta análisis a partir de la literatura de los 12 años previos sobre terapia grupal de adultos (116 trabajos), y encontraron que, efectivamente, la terapia grupal sí obtiene resultados positivos. Otros estudios previos sostienen que los tratamientos grupales son igualmente eficaces que los individuales $(3,4,5,6,7,8)$.

Centrándonos más en la población que nos ocupa, Hoag y Burlingame (9) en su trabajo de 1997 tomaron 56 estudios sobre terapia grupal con niños y adolescentes y realizaron un estudio de meta análisis encontrando índices de efi- 
cacia similares a los hallados en adultos; en la mayoría de los estudios que revisaron, los pacientes mejoraban más en los grupos terapéuticos que los controles, ya fueran listas de espera o grupos placebo. Igualmente, estos autores señalan que diferentes diagnósticos no implican diferentes respuestas al tratamiento grupal; todos los diagnósticos investigados se favorecían de él en el mismo grado.

Este mismo estudio apoya también la mayor eficacia de los grupos terapéuticos en contexto clínico, respecto a aquellos que se aplican en otros contextos, en contra de los que previamente habían encontrado otros trabajos, que no hallaron diferencias (10). Este dato, a propósito de la supremacía del contexto clínico, tiene una relevancia especial como argumento a favor de la eficacia de los tratamientos grupales, ya que los sujetos incluidos en estudios efectuados en contextos clínicos suelen presentar mayor patología que los tomados de otros contextos, a menudo muestras subclínicas.

En esta misma línea, otro trabajo sobre grupos terapéuticos con estudiantes de instituto (11) muestra que los tratamientos grupales son eficaces en el abordaje de trastornos graves como la depresión, y que esta eficacia no está relacionada con la orientación del grupo (se compararon la terapia cognitiva y la terapia del proceso interpersonal con iguales resultados).

Finalmente, y yendo más allá, otros autores se han planteado si la experiencia del grupo terapéutico podría tener beneficios añadidos al de la mejoría psicopatológica. Así, los diversos estudios de Shechtman con estudiantes israelíes señalan que el paso por un grupo terapéutico en la adolescencia tiene efectos sobre su autoestima, y legitima de cara a los pacientes una comunicación a un nivel más profundo, sirviendo de práctica para sus relaciones sociales, y favoreciendo las amistades íntimas dentro y fuera del grupo $(12,13,14,15)$.

El presente trabajo describe la experiencia de tratamiento grupal con adolescentes y jóvenes desarrollada en un Centro de Salud Mental a través del trabajo con tres grupos consecutivos llevados a cabo entre los años 2002 y 2005.

\section{Método}

La orientación teórica que guió la intervención fue la de grupo operativo (16), con una periodicidad semanal. El número total de pacientes atendidos fue de 43, estando los grupos formados entre 14 y 17 participantes, siendo un $28 \%$ varones y un $72 \%$ mujeres (media de la edad $=18,57$; desviación típica $=1,59$ ) (Figura 1). 
Figura 1

Distribución de la edad de los participantes

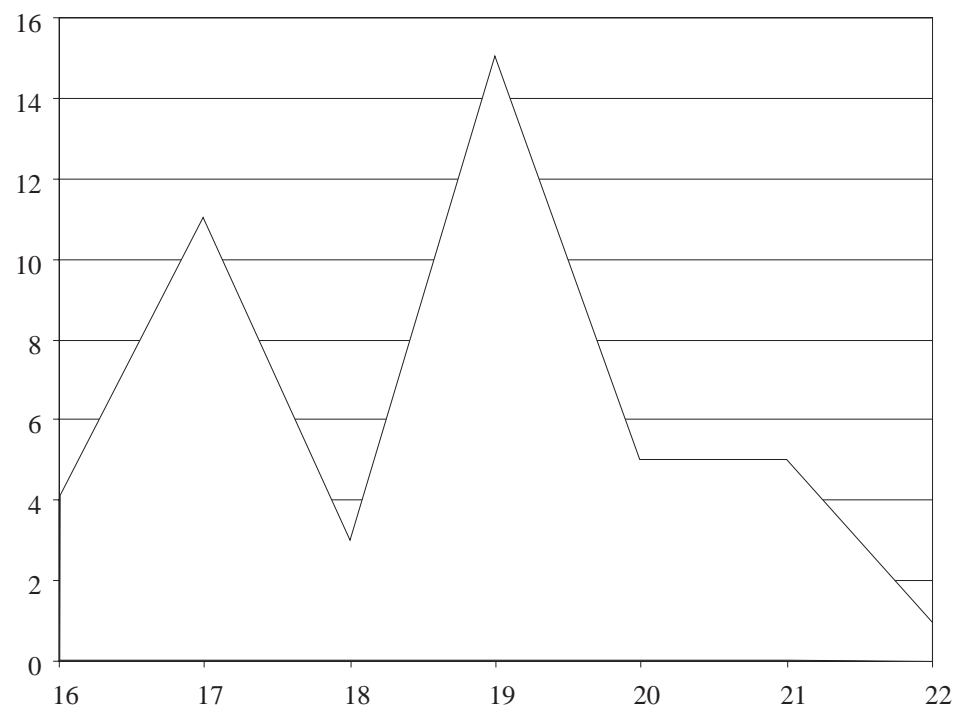

Respecto al origen de la derivación al grupo terapéutico, el $65 \%$ de los pacientes eran derivados por psicólogo clínico mientras que el 35\% restante eran derivados por psiquiatra y recibían tratamiento farmacológico previamente a su incorporación al grupo. La distribución de sus diagnósticos (según DSM-IV-TR) (17) y la duración del tratamiento previo a la intervención grupal se recogen en las figuras 2 y 3.

Para la recogida de información se ha empleado un protocolo que recoge datos acerca de la clínica previa al tratamiento, la situación actual y la valoración subjetiva de su participación, así como una medida pre y post-tratamiento del SCL-90-R. La escala de síntomas, SCL-90-R (18), nos permite una evaluación general de síntomas psicopatológicos. Es un listado de comprobación de síntomas recogidos en 9 dimensiones con datos normativos españoles y propiedades psicométricas aceptables (19). En cuanto a la fiabilidad, ha presentado una buena consistencia interna en todas las subescalas (oscilando la $\alpha$ de Cronbach de 0,78 a 0,90), así como una adecuada validez predictiva (20). También ha demostrado adecuada validez discriminativa, la validez concurrente con el IIP-C y el GHQ-12 (21).

Los objetivos trabajados en el grupo fueron los siguientes: Adquisición de su propia identidad. La identificación con otras personas es un aspecto fundamental 
Figura 2

Distribución de los diagnósticos de los participantes

\begin{tabular}{|c|c|c|c|c|}
\hline $\begin{array}{c}\text { Tr. de la Alimentación } \\
25 \%\end{array}$ & Tr. Adaptativos & Tr. de Ansiedad & $\begin{array}{c}\text { Tr. de estado de } \\
\text { Ánimo } \\
16 \%\end{array}$ & $\begin{array}{c}\text { Tr. de } \\
\text { Personalidad } \\
9 \%\end{array}$ \\
\hline
\end{tabular}

Figura 3

Duración del tratamiento previo a la intervención grupal

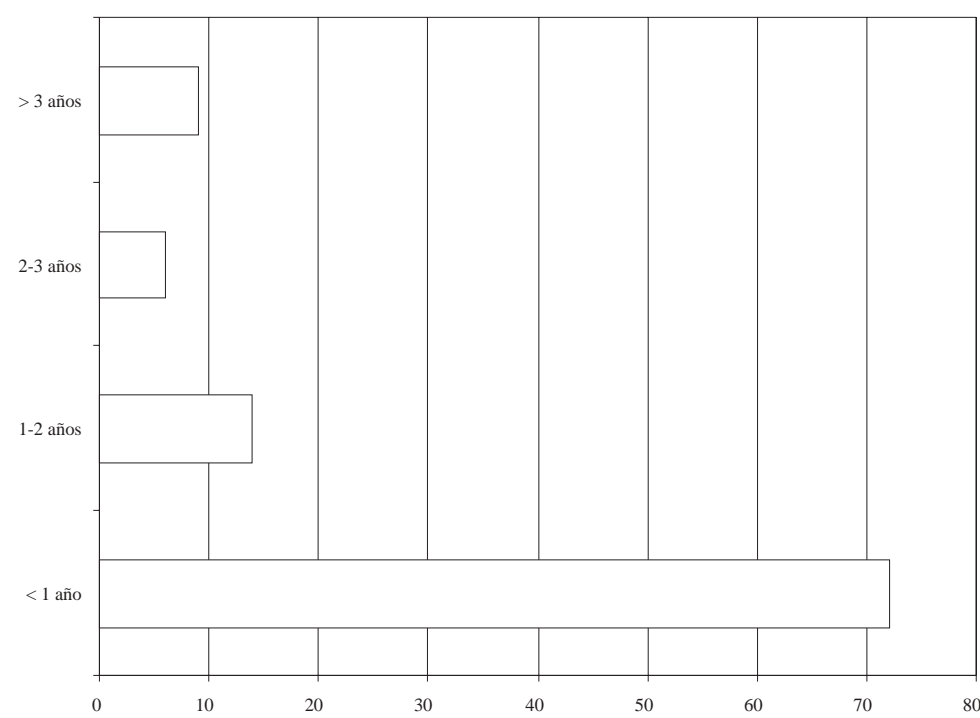

en la adolescencia (22). El continuo cambio de roles que supone hacerse adulto conlleva un esfuerzo para redefinir lo que son, y la búsqueda de un equilibrio entre lo que pueden rescatar de su infancia para constituir el futuro, y las nuevas actitudes, responsabilidades y formas de sentir propias del mundo de los adultos. Una de las vías para lograrlo es la identificación con personas relevantes que puedan servir de modelos. La ausencia de referentes positivos entre los adultos puede complicar esta identificación. Poder rescatar lo positivo de estas relaciones, y, en general, prestar atención a este proceso es uno de los objetivos.

Reflexionar sobre las relaciones con los padres, así como la historia familiar y su implicación en ella (nuevas formas de convivencia). Estos nuevos roles pue- 
den traer consigo nuevos conflictos en las relaciones familiares (23), que tienen que redefinirse según las nuevas demandas de los padres y las nuevas necesidades de los hijos. Entre los propósitos del grupo está verbalizar las dificultades que están encontrando en las nuevas formas de convivencia (padres separados, familias reconstruidas, padres ausentes, etc.) y analizar su nivel de implicación en los conflictos que se presentan.

Aceptación de la imagen corporal, desarrollo de su identidad sexual y organización sexual definitiva. La sexualidad es otra de las áreas importantes a trabajar (24). Las primeras experiencias sexuales y el sentido que cobran como nuevo medio de relación son vitales y suponen un aspecto definitorio de la propia identidad. Con relación a esto y en vista de las patologías que presentan los pacientes del grupo, otro foco de interés es el de la aceptación de la imagen corporal.

Reflexionar y verbalizar las dificultades en las relaciones afectivas y con los iguales. Otra área que para los adolescentes cobra especial importancia es la de las relaciones sociales y afectivas (25), en las que encuentran apoyo y nuevos referentes de comportamiento, y que al estar muy cargadas emocionalmente conllevan muchos conflictos. Poder definir estas relaciones en términos positivos, aprender nuevas formas de comunicarse y reflexionar sobre las situaciones de lealtad, coherencia y confianza en los demás es otro de los objetivos del grupo.

Favorecer su integración social. Se busca facilitar la incorporación a la vida adulta, la integración social, y la satisfacción personal (mundo laboral, futuro, etc.).

\section{Resultados}

Mientras que el 65,9\% de los pacientes fueron dados de alta después de la intervención grupal (31,8\% recibieron el alta por fin de tratamiento y el 34,1\% fueron altas voluntarias o abandonos), el 34,1\% restante continúa en tratamiento en nuestro Centro de Salud Mental. La situación académica de los pacientes después del tratamiento es: $23 \%$ progresa adecuadamente, $31 \%$ progresa con dificultades, $23 \%$ interrumpe voluntariamente sus estudios, $8 \%$ presenta un fracaso escolar y $15 \%$ no estudia.

La gran mayoría de los pacientes (77\%) valoran la experiencia de participar en el grupo como buena y el $23 \%$ como regular. Respecto al nivel de satisfacción con la intervención, $15 \%$ está muy satisfecho, $77 \%$ satisfecho, y $8 \%$ poco satisfechos.

El perfil de puntuaciones en el SCL-90-R manifiesta una disminución de la intensidad en todas las escalas de psicopatología después de la intervención gru- 
pal. Sin embargo, estas diferencias no alcanzaron significación estadística tras aplicar una prueba t, posiblemente por el tamaño muestral. Como se aprecia en la tabla, las diferencias más importantes aparecieron en las dimensiones que evalúan depresión, ansiedad y ansiedad fóbica.

Tabla 1

Perfil de puntuaciones en el SCL-90-R antes y después de la intervención grupal

\begin{tabular}{|l|c|c|c|c|}
\hline \multirow{2}{*}{} & \multicolumn{2}{|c|}{ Pre- } & \multicolumn{2}{c|}{ Post- } \\
\cline { 2 - 5 } & Media & D. T. & Media & D. T. \\
\hline Somatización (0-4) & 1,47 & 0,86 & 1,06 & 0,90 \\
\hline Obsesivo-compulsiva (0-4) & 1,87 & 0,84 & 1,56 & 0,80 \\
\hline $\begin{array}{l}\text { Susceptibilidad } \\
\text { interpersonal (0-4) }\end{array}$ & 1,95 & 0,86 & 1,57 & 1,16 \\
\hline Depresión (0-4) & 2,24 & 0,90 & 1,69 & 1,09 \\
\hline Ansiedad (0-4) & 1,84 & 0,85 & 1,50 & 0,90 \\
\hline Hostilidad (0-4) & 1,60 & 1,05 & 1,25 & 0,95 \\
\hline Ansiedad fóbica (0-4) & 1,37 & 0,72 & 0,90 & 0,54 \\
\hline Ideación paranoide (0-4) & 1,64 & 0,83 & 1,42 & 1,01 \\
\hline Psicoticismo (0-4) & 1,17 & 0,66 & 0,97 & 0,70 \\
\hline $\begin{array}{l}\text { Índice de } \\
\text { Severidad Global (0-4) }\end{array}$ & 1,69 & 0,62 & 1,32 & 0,73 \\
\hline $\begin{array}{l}\text { Índice de Malestar } \\
\text { Referido a los Síntomas } \\
\text { Positivos (0-4) }\end{array}$ & 5,46 & 2,77 & 4,07 & 2,69 \\
\hline Índice de Síntomas Positivos & 62,97 & 15,51 & 53,11 & 22,73 \\
\hline
\end{tabular}

Finalmente, respecto a la valoración que los pacientes hacían a la finalización del grupo acerca de los aspectos mejorados después de la intervención, los más destacados fueron: la disminución de la inseguridad y la sensación de aislamiento, el aprendizaje de recursos de autocontrol, la reducción de su nivel de ansiedad, el incremento de capacidad de introspección y de la toma de conciencia de sus propias dificultades, el afrontamiento activo de sus miedos y preocupaciones, y el poder reafirmarse sobre sus proyectos y metas de futuro. Asimismo, la gran mayoría de ellos sostuvieron que el grupo les había permitido sentirse aceptados y seguros, y que poder compartir sus problemas con iguales había mejorado su comunicación con los demás tanto dentro como fuera del grupo. 


\section{Discusión}

En relación con los contenidos que aparecen en las sesiones de terapia, los motivos de consulta expresados por ellos suelen ser la ansiedad, baja autoestima, problemas con la alimentación, dificultades en la aceptación de su cuerpo, problemas familiares en los que los jóvenes están implicados y/o se involucran, dificultades en las relaciones con sus iguales y relaciones afectivas. También su futuro y cómo se van incorporando al mundo adulto es otro motivo de duda e insatisfacción.

El grupo aparece como el lugar donde poder rescatar su pasado, sus vivencias, y poder hablar de ello, elaborarlo con un nivel de maduración y comprensión mayor. En ocasiones parecen absortos en su mundo, aislados en sus reflexiones, los silencios son frecuentes, suponen un tiempo de elaboración y de diferenciación con el grupo.

El sentimiento de dependencia-independencia, el temor y la defensa frente a él, característicos de estas edades, también está presente en el grupo a través de las ausencias e incorporaciones de nuevo y de los abandonos. Es un lugar de paso con tiempos de permanencia, en algunos casos, breves, pero les ayuda a ir encauzando su vida y observamos con mucha frecuencia que este abandono del tratamiento grupal no va unido a una nueva demanda de atención en Salud Mental.

Hemos agrupado los contenidos verbalizados de una manera predeterminada, en bloques temáticos que nos parecen importantes, ya que están relacionados con los objetivos trabajados en el grupo. Dichos bloques temáticos son: identidad propia, relación con los padres y con la familia en general, relaciones afectivas o de pareja, sexualidad y vivencias relacionadas con el propio cuerpo, amistad y relación con los iguales, integración social e incorporación a la vida adulta, su vivencia sobre la enfermedad y, por último, verbalizaciones relacionadas con la muerte.

\section{Propia identidad}

En las sesiones verbalizan sentimientos de inseguridad, duda, dependencia de los demás, se comparan con ellos, se exigen a sí mismos y esto les genera insatisfacción, dificultad para aceptarse como son, sentimientos de rareza, de ser diferentes a los demás y desconfianza. En ocasiones es una identidad forjada a partir de los deseos de los otros, una falsa identidad y se sienten perseguidos o con temor de ser dañados. Comienzan también a reafirmarse en sus criterios propios, a diferenciarse de los demás, a reflexionar sobre el esfuerzo y las pérdidas que supone su maduración. La frustración es difícilmente admitida. Algunos ejemplos son: «yo me veo patética», «soy muy exigente, muy radical... no me veo capaz», «toda mi vida ha sido como una obra de teatro, actuando para que los demás estén a gusto y no yo», «siento que todo el mundo me quiere hacer daño, que se van a 
aprovechar de mí, tengo mucha desconfianza con todo el mundo», «no me encuentro bien y no me acepto como soy», «estoy quemada, asqueada y mal».

\section{Relaciones con los padres y familia}

Hay contenidos relacionados con el conflicto edípico, rivalidad con la madre, etc., pero también aparecen situaciones traumáticas, separaciones de los padres y reconstrucción con nuevas parejas, hermanos no conocidos, necesidad de reencuentro con el padre o madre ausente y la posibilidad de reelaboración de vivencias o realidades de abandono. Verbalizan experiencias de agresiones sexuales, maltrato y dinámicas familiares conflictivas, donde ellos se sienten y/o están implicados e intentan solucionar los problemas. Son contenidos como: «no aguanto a mi madre y por eso estoy aquí... mi madre es muy protectora, no me deja vivir, me trata como a una niña», «mi madre es la mujer a la que no quiero parecerme, va de dura», «discutí con mi padre y no me pidió perdón... ahora sueño con mi padre que regresa por no quererle... nos maltrataba, pero se puso malo y yo no estaba con él» (los padres de esta joven están separados, ella nos cuenta en la octava sesión que su padre murió hace un año y no pudo estar ni despedirse de él), «a los diez años me sentía sola, mi padre me encerraba y se llevaba a mi hermana. Mi madre no hacía nada, no lo hacía por no quedarse sola... yo tenía sentimientos de vacío», «tengo miedo del rechazo de mi madre y por eso no quería buscarla... me hago preguntas... ¿ ¿por qué me abandonó?, ¿por qué tiene otros niños y a mí no?» (su madre la abandona ocho días después del nacimiento, es criada por los abuelos paternos).

Relaciones afectivas o de pareja

En la adolescencia se produce un cambio en la relación con los objetos edípicos. Los deseos edípicos son experimentados dentro del contexto de una persona sexualmente madura y el joven encuentra una solución de compromiso entre lo que es deseado y lo que puede ser permitido, busca gratificación a través de nuevos objetos y ello configura su identidad sexual adulta. Hay también un cambio en la relación con los iguales. Verbalizan sus dificultades en estos aspectos y también en compaginar su relación de pareja con la relación con los amigos/as, esto provoca la ruptura en muchas ocasiones. El otro/a es para ellos un punto de apoyo importante, les ayuda en su reafirmación y les da estabilidad. A veces muestran sentimientos de celos, desconfianza y proyectan su propia agresividad y frustración en su pareja. Manifiestan su dependencia hacia él/ella y son frecuentes las autolesiones o gestos autolíticos después de una discusión o ruptura de la relación. Comentan «antes tenía estabilidad por tener novio... hay que encontrar a la persona adecuada», «quiero un hombre parecido a mi padre, que sea afín con el carác- 
ter de él», «yo estaba mal con el otro novio, quería estar con mis amigas... necesito estar sola, no sé por qué he dejado a mi novio», «yo ocupo un lugar especial con mi padre, soy su niña... nadie me va a querer como hombre más que mi padre», «yo todo lo pago con mi novio».

\section{Sexualidad y propio cuerpo}

El desarrollo principal en la adolescencia es el establecimiento de la organización sexual definitiva y la adquisición de la propia identidad sexual como varón o hembra. El cuerpo ocupa un lugar central, tanto en su propia estima narcisista, como en el sentimiento de aprobación y aceptación por parte del otro. A veces existe una visión distorsionada del propio cuerpo, con sentimientos de odio y vergüenza, que refleja sus miedos e inseguridades. Hablan de un cuerpo del que se defienden, expuesto a la mirada del otro, que les provoca sentimientos de fragilidad e inseguridad, necesitan tener una imagen corporal aceptable, se comparan con los otros y se exigen a sí mismos. De ellos mismos expresan: «he cortado con un chico y ahora me arrepiento, me siento vacía, estaba confundida, me da miedo el sexo... miedo al momento del sexo, miedo y vergüenza», «ahora me siento más mujer y no tan niña, ahora estoy empezando a vivir, no quiero sufrir tanto», «yo puedo ser exigente, tener un cuerpo 10», «no me gusto a mí mismo, por la autoestima, no me caigo muy bien, no me gusta mi cuerpo», «yo me veo ridículo, patético, por la forma de ser amariconado».

\section{Amistades}

La amistad y la relación con los iguales adquieren gran importancia, los compañeros del mismo sexo le sirven como objeto de identificación y los del sexo opuesto, como objeto de sus deseos sexuales. Hay una oscilación entre los sentimientos de dependencia e independencia. La lealtad y sinceridad son valores importantes. En esta etapa se produce la diferenciación en relación con el grupo de iguales y la adquisición de su propia identidad. Algunos contenidos son: «me dejó mi novio... tengo problemas con los amigos y no tengo con quien salir. No tengo nada. Son muy falsos, no soy su amiga en realidad», «tienes que gustarte a ti misma y ya está... mi amiga me copia en todo y la dejé... no te ha demostrado que es tu amiga, tienes que hacerla el vacío», «tengo una amiga que ha cambiado mucho, ahora me machaca y me pone en evidencia, es mi amiga, pero me molesta que cambie y sea como una modelo», «me cuesta relacionarme».

Integración social e incorporación a la vida adulta

Aparece el miedo a fracasar y la angustia que les despierta, miedo a elegir y a equivocarse y dificultades para integrarse en la vida adulta. El trabajo es un lugar 
de reconocimiento y afirmación. Expresiones como: «he dejado de estudiar y duermo tranquila, ahora busco trabajo» (repetía por tercera vez el curso), «tengo muchos proyectos y no me doy atracones... también he crecido, no puedo quedarme en el pasado», «estoy perdida, he pensado dejar la carrera, me agobia... estoy cansada de todo», «he tenido que dejar el trabajo, me dio un ataque de ansiedad, sólo pienso que me voy a morir», «ahora pienso en los estudios y me da miedo, también me da por pensar que no sirvo para nada, miedo al trabajo y miedo a quedarme sola en la vida».

Vivencias sobre la enfermedad

En ocasiones la enfermedad les da identidad. Expresan ansiedad, duda, miedo y dificultad para afrontar las situaciones que les plantea la vida y sentirse bien consigo mismos. El cuerpo refleja sus problemas a otros niveles, no comer como desplazamiento de otro conflicto. Existen en muchos casos una realidad familiar problemática (separaciones traumáticas, alcoholismo, discusiones frecuentes, etc.) y el nivel de implicación de estos jóvenes es alto, mediando en dichos problemas, sobre todo en la conflictiva entre los padres. En otros momentos hablan de su dificultad para expresar lo que les ocurre. Comentan en las sesiones «soy una bulímica», «no sé lo que me pasa, soy infeliz, sólo cuento los síntomas», «estoy aquí por crisis de ansiedad, cambio de domicilio, he roto con mi novia, soy muy nervioso», «vomitar es una felicidad prestada, es mi forma de vida. No sé si quiero salir. Me siento sola. Es una adicción, me lleno, me ahoga esa sensación, me doy asco y lástima, si me vacío, me siento mejor», «tengo miedo de salir de todo esto, miedo de mí misma».

\section{Verbalizaciones relacionadas con la muerte}

La adolescencia significa pérdida y muerte. Pérdida del niño y paso a la edad adulta, pérdida de los padres idealizados, miedo al futuro, a la soledad y a afrontar su propia vida. Expresan su dificultad para elaborar el duelo por familiares y es a partir de su enfermedad y del tratamiento grupal que pueden hablar de ello. En muchos casos, esos familiares han sido importantes figuras de apego (abuelos sobre todo). Las autolesiones y los gestos autolíticos son frecuentes, secundarios a discusiones familiares o rupturas de pareja. Hay una baja tolerancia a la frustración, fragilidad emocional y angustia ante el fracaso. Sus formas de expresarlo son: «no he superado la muerte de dos abuelos, no puedo expresar las emociones... el que llora, se desahoga, ¿pero el que no?», «lloro por todo, estoy muy sensible, me pongo como loca y me pongo a cortarme, me veo sangrando y paro... cuando me veo llena de sangre, me quedo como relajada», «no quiero hacer daño a mis padres y decirle a mi madre que me quiero morir, pero lo pienso y no lo 
hago, me doy alternativas», «una amiga se suicidó, yo pensé que me iba a morir y todo el mundo se va a morir», «se murió mi abuela hace un año y me acuerdo de ella, sueño mucho con mi abuela y estoy mal por cualquier cosa».

A lo largo de las experiencias grupales que se han realizado, vemos cómo los jóvenes entienden el grupo como un lugar donde desahogarse y quejarse, donde pueden llevarles la contraria, que ayuda a pensar, es un espacio «democrático» en el que los demás ven los problemas desde fuera y dan su opinión.

Desde nuestra visión crítica como terapeutas, observamos que el grupo se constituye con pacientes de diferentes edades (franja etaria desde los 16 a 22 años), donde la problemática que se plantea es diferente. En los adolescentes lo importante son temas relacionados con el conflicto edípico, dificultades de aceptación del esquema corporal, sexualidad y relación con los iguales. Los jóvenes o adolescentes tardíos, verbalizan sus dificultades de integración en el mundo adulto, problemas laborales, de pareja, conflictos familiares en los que ellos están implicados y problemas relacionados con sus rasgos de personalidad.

El encuadre debe ser recordado en diferentes momentos, sobre todo en el inicio y en cuestiones referentes a las relaciones interpersonales. Desarrollan a partir del grupo de terapia relaciones de amistad o de ayuda, se implican en los problemas de los demás y aparecen conflictos de lealtad y sobreimplicación. Esto podría modificar el objetivo del tratamiento y dejar vacío de contenidos el espacio grupal.

Los abandonos son frecuentes y en algunos momentos del desarrollo del grupo, interfieren u obstaculizan la marcha del mismo (26). Los que permanecen no entienden la falta de compromiso de los que abandonan y existe un sentimiento de frustración que es necesario abordar junto con la elaboración de la pérdida. Sin embargo, el sentimiento de permanencia, tan importante en esta etapa vital, está presente en el grupo. Los integrantes participan de una manera comprometida, dando continuidad y cohesión al espacio grupal y utilizando este encuadre como un lugar de cooperación y elaboración, siendo una experiencia útil y positiva para ellos en general.

\section{Conclusiones}

El período de la adolescencia y juventud supone un proceso gradual de maduración, aporta una contribución específica y esencial en la construcción del yo. Los trastornos psíquicos en este período deben entenderse de una manera diferente a los que se presentan en la infancia y la vida adulta.

El grupo de terapia en los adolescentes y jóvenes es vivido como un lugar de apoyo, encuentro, pertenencia y de contención en algunos casos. Es el lugar donde 
pueden expresar sus dificultades en este proceso y sus vivencias de soledad, ansiedad, inseguridad y miedo al fracaso. Verbalizan sus dificultades en el afrontamiento de la realidad de la vida adulta. Ayuda a disminuir la autoexigencia y facilita la comprensión de conflictos no resueltos que influyen en su desarrollo actual y futuro. Favorece la introspección y la posibilidad de compartir con los demás sus experiencias, incrementando el sentimiento de seguridad y aceptación por parte del grupo. Es, finalmente, un lugar de apoyo al que poder acudir, pero también abandonar para desarrollar sus propias experiencias y proyectos personales como adultos.

\section{BIBLIOGRAFÍA}

(1) LAUFer, M.; LAUfER, E., La adolescencia y crisis del desarrollo, Barcelona, Espaxs, 1988.

(2) Burlingame, G. M., y otros, «Group Psychotherapy Efficacy: a Meta-analytic Review», en Funriman, A., Group Psychotherapy Efficacy: a Meta-analytic Perspective, Simposio de la Asociación Psicológica Americana, Nueva York, 1995.

(3) WeIsz, J. R., y otros, «Effectiveness of Psychotherapy with Children and Adolescents: a Metaanalysis for Clinicians», Journal of Consulting and Clinical Psychology, 55, 1987, pp. 542-549.

(4) BAER, R. A.; NIETZEL, M. T., «Cognitive and Behavioural Treatment of Impulsivity in Children: a Meta-analytic Review of the Outcome Literature», Journal of Clinical Child Psychology, 20, 1991, pp. 400-412.

(5) Roberts, A. R.; Camasso, M. J., «The Effects of Juvenile Offender Treatment Programs of Recidivism: a Meta-analysis of 46 Studies», Notre Dame Journal of Law, Ethics \& Public Policy, 5, 1991, pp. 421-441.

(6) Russell, R. L.; Greenwald, S.; SHIRK, S. R., «Language Change in Child Psychotherapy: a Meta-analytic Review», Journal of Consulting and Clinical Psychology, 59, 1991, pp. 916-919.

(7) Grossman, P. B.; HugheS, J. N., «Self-control Interventions with Internatializing Disorders: a Review and Analysis», School Psychology Review, 21, 1992, pp. 229-245.

(8) SHIRK, S. R.; Russell, R. L., «A Reevaluation of Estimates of Child Therapy Effectiveness», Journal of American Academy of Child and Adolescent Psychiatry, 31, 1992, pp. 703-709.

(9) Hoag, M. J.; Burlingame, G. M., «Evaluating the Effectiveness of Child and Adolescent Group Treatment: a Meta-analytic Review», Journal of Clinical Child Psychology, 26, 1997, pp. 234-246.

(10) WeIss, B; WeIsz, J. R., «The Impact of Methodological Factors on Child Psychotherapy Outcome Research: a Meta-analysis for Researchers», Journal of abnormal Child Psychology, 18, 1990, pp. 639-670.

(11) Hogg, J. A.; Deffenbacher, J. L., «A Comparison of Cognitive and Interpersonal Process Group Therapies in the Treatment of Depression Among College Students», Journal of Counselling Psychology, 35, 1988, pp. 304-310.

(12) Shechtman, Z., «Small Group Therapy and Preadolescent Same Sex Friendship», International Journal of Group Psychotherapy, 41, 1991, pp. 227-243.

(13) Shechtman, Z., «Group Psychotherapy for the Enhancement of Intimate Friendship and Self-esteem Among Troubled Elementary School Children», International Journal of Group Psychotherapy, 10, 1993, pp. 483-494. 
(14) Shechtman, Z., «Group Counselling/Psychotherapy as a School Intervention to Enhance Close Friendships in Preadolescence», International Journal of Group Psychotherapy, 44, 1994, pp. 377-391.

(15) Shechtman, Z.; Vurembrand, N.; HertZ-Lazarowitz, R., «A Dyadic and Gender-specific Analysis of Close Friendships of Preadolescents Receiving Group Psychotherapy», Journal of Social and Personal Relationships, 11, 1994, pp. 443-448.

(16) PichON-RIvière, E., El proceso grupal. Del psicoanálisis a la psicología social, Buenos Aires, Nueva Visión, 1985.

(17) American Psychiatric Association, DSM-IV-TR. Manual diagnóstico y estadístico de los trastornos mentales, Barcelona, Masson, 2002 (texto revisado).

(18) Derogatis, L., SCL-90-R: Administration, Scoring and Procedure Manual. Baltimore, Clinical Psychometric Research, 1977.

(19) GonZÁlez De Rivera, J. L., y otros, The Spanish Version of the SCL-90-R. Normative Data in the General Population, Towson, Clinical Psychometric Research, 1989.

(20) Schmitz, N., y otros, «The Symptom Check-List-90-R (SCL-90-R): A German Validation Study», Quality of Life Research, 9, 2000, pp. 185-193.

(21) Schmitz, N., y otros, «Diagnosing Mental Disorders in Primary Care: the General Health Questionnaire (GHQ) and the Symtom Check List (SCL-90-R) as Screening Instruments», Social Psychiatric Epidemiology, 34, 1999, pp. 360-366.

(22) LAUFER, M., El adolescente suicida, Madrid, Biblioteca Nueva, 1998.

(23) Winnicott, D. W., La familia y el desarrollo del individuo, Buenos Aires, Hormé, 1995.

(24) LASA, A., «Experiencias del cuerpo y construcción de la imagen corporal en la adolescencia: vivencias, obsesiones y estrategias», Psicopatología y Salud Mental del niño y del adolescente, 2, 2003, pp. 53-74.

(25) LILLO, J. L., «Crecimiento y comportamiento en la adolescencia», Revista de la AEN, 9 , 2004, pp. 57-71.

(26) Bion, W. R., Experiencias en grupos, Barcelona, Paidós, 1990.

* Pilar Sánchez del Hoyo, psicóloga Clínica; Luis Javier Sanz Rodríguez, psicólogo clínico; Concepción Baro Santamarta; María Gómez García de la Pedrosa, P.I.R. Hospital Universitario de Getafe. Todos pertenecen a los Servicios de Salud Mental de Parla (Madrid). Correspondencia: Luis Javier Sanz Rodríguez. C/ Mayor, 23. 28991 (Madrid). ljsanz@cop.es 